\title{
Risk factors and in-hospital mortality in Chinese patients undergoing coronary artery bypass grafting: Analysis of a large multi-institutional Chinese database
}

\author{
Zhe Zheng, MD, ${ }^{\mathrm{a}}$ Lu Zhang, MD, ${ }^{\mathrm{a}}$ Shengshou Hu, MD, ${ }^{\mathrm{a}} \mathrm{Xi} \mathrm{Li}, \mathrm{MD},{ }^{\mathrm{b}}$ Xin Yuan, MD, ${ }^{\mathrm{a}}$ and \\ Huawei Gao, MD, ${ }^{\text {a }}$ for the Chinese Cardiovascular Surgical Registry Study
}

Objective: This study was undertaken to delineate outcomes and to assess risk factors for in-hospital mortality among Chinese patients undergoing coronary artery bypass grafting.

\begin{abstract}
Methods: From 2007 to 2008, a total of 9838 consecutive adult patients undergoing coronary artery bypass grafting were enrolled in the Chinese Coronary Artery Bypass Grafting Registry, which included 43 centers from 17 province-level regions in China. This registry collected information on 67 preoperative factors and 30 operative factors believed to influence in-hospital mortality. The relationship between risk factors and inhospital mortality was evaluated by univariate and logistic regression analyses.
\end{abstract}

Results: Overall in-hospital mortality was $2.5 \%$. Eleven risk factors were found to be significant predictors for outcome: age (continuous), body mass index (continuous), left ventricular ejection fraction (continuous), preoperative New York Heart Association functional class III or IV, chronic renal failure, extracardiac arteriopathy, chronic obstructive pulmonary disease, preoperative atrial fibrillation or flutter (within 2 weeks), preoperative critical state, other than elective surgery, and combined valve procedure. Calibration with the HosmerLemeshow test was satisfactory $(P=.35)$, and the discrimination power was good (area under the receiver operating characteristic curve, $0.81 ; 95 \%$ confidence interval, $0.79-0.84)$.

Conclusions: The risk profiles and in-hospital mortality of Chinese patients undergoing coronary artery bypass grafting were determined from data in the most up-to-date multi-institutional database. Eleven variables were demonstrated to be independent risk factors for in-hospital death after coronary artery bypass grafting. ( $\mathrm{J}$ Thorac Cardiovasc Surg 2012;144:355-9)

Supplemental material is available online.

China has recently witnessed a dramatic increase in the incidence of coronary artery disease, a phenomenon known as the epidemiologic transition. ${ }^{1}$ To meet the rapidly growing

\footnotetext{
From the Department of Cardiovascular Surgery, ${ }^{a}$ State Key Laboratory for Cardiovascular Disease, Fuwai Hospital, National Center for Cardiovascular Disease, Peking Union Medical College and Chinese Academy of Medical Sciences, Beijing, China; and the Department of Epidemiology and Biostatistics, ${ }^{\mathrm{b}}$ School of Public Health, Peking University Health Science Center, Beijing, China.

Supported by Public Specialty Fund of Health Ministry (200902001), the Key Project in the National Science and Technology Pillar Program during the Eleventh 5-Year Plan Period (2006BAI01A09), and the Program for Changjiang Scholars and Innovative Research Team in University Basic Scientific Research Fund of National Public Scientific Institute.

Disclosures: Authors have nothing to disclose with regard to commercial support. Z.Z. and L.Z. contributed equally to this article as first coauthors.

Dr Lu Zhang's current affiliation is Department of Medicine, Peking Union Medical College Hospital, Peking Union Medical College and Chinese Academy of Medical Sciences, Beijing, China.

Received for publication April 18, 2011; revisions received Sept 17, 2011; accepted for publication Oct 4, 2011; available ahead of print Nov 7, 2011.

Address for reprints: Shengshou Hu, MD, 167A Beilishilu, Beijing 100037, The People's Republic of China (E-mail: shengshouhu@yahoo.com).

0022-5223/\$36.00

Copyright (c) 2012 by The American Association for Thoracic Surgery

doi:10.1016/j.jtcvs.2011.10.012
}

demand of patients who need revascularization with coronary artery bypass grafting (CABG), Chinese institutions and surgeons have elected to carry out increasing numbers of CABG procedures. With little knowledge of the current risk profiles of Chinese patients undergoing CABG, however, crude mortality rates have often been used as an indicator for quality of care in China, which might bring considerable bias in quality comparisons among institutions and might even interfere with providing surgical service for high-risk patients who could gain most from surgical treatment, despite increased risk. ${ }^{2}$ We embarked on this study with the intent of establishing the risk profile of adult patients undergoing CABG in China and to determine the in-hospital mortality among these patients. This study is based on the most up-to-date multi-institutional Chinese database. This effort is especially important because recent literature has revealed that conclusions drawn from Western databases (eg, EuroSCORE) might not be suitable for this cohort of patients. ${ }^{3}$

\section{MATERIALS AND METHODS \\ Database Setup}

The Chinese CABG Registry is a national multi-institutional study with the aim of exploring the risk factors and clinical outcomes for Chinese patients undergoing CABG. Its steering group was set up to include a number 


\section{Abbreviation and Acronym}

$\mathrm{CABG}=$ coronary artery bypass grafting of Chinese cardiac surgeons and epidemiologists with an interest in the field of CABG surgical risk from Fuwai Hospital. Forty-three participating institutions from 17 province-level regions in mainland China (there are 31 province-level regions in mainland China, including 22 provinces, 4 directly controlled municipalities, and 5 autonomous regions, excluding Hong Kong, Macau, and Taiwan) enrolled a total of 9838 adult patients undergoing CABG from January 2007 to December 2008, with data prospectively collected (Figure 1). Because of the economic imbalance in mainland China, most of the cardiac centers that were capable of carrying out CABG procedures were located in the eastern part of the country, as shown by the participating centers of our registry (Figure 1). Patients from all over mainland China (all 31 province-level regions, plus patients from Macau) received treatment provided by institutions of our registry, however, which enabled our database to be representative of the whole Chinese population (Figure 2). Detailed information on patient demographic characteristics, preoperative risk factors, operative risk factors, and in-hospital mortality were collected. The data collected for the registry included 67 preoperative factors (Appendix E1) and 30 operative parameters (Appendix E2) that were selected on the basis of our clinical experience previous published literature concerning the risk factors for $\mathrm{CABG}$ procedures.

\section{Quality Control of Data}

Two reviewers from the data processing center abstracted a random sample of $5 \%$ to $10 \%$ of medical records through on-site auditing at a 6-month interval. The data submitted by the hospitals were compared with information in the medical records. When disagreement occurred, a physician adjudicated the disagreement to determine the final value. This final database, referred to as the Chinese Cardiovascular Surgical Registry database, was then scrutinized carefully. A total of 274 patients were found to have incomplete data records: of them, 5 patients were without listed age, 70 without listed weight, 52 without listed height, 205 without listed ejection fraction values, 1 without data regarding unstable angina, 2 without data regarding cardiogenic shock, and 80 without listed New York Heart Association functional class. Some of these patients had more than 1 missing data field. Considering the fact that they only accounted for a very small proportion of our patients, we imputed missing continuous variables (age, weight, height, and ejection factor values; for weight and height, different mean values were used for the sexes) with mean values and missing categoric variables with negative values (unstable angina, cardiogenic shock, New York Heart Association stage) so that patients who might have experienced the end point (in-hospital death) would not be excluded from analysis simply for 1 or 2 missing variables among the many examined.

\section{Statistical Analysis}

Statistical analysis was performed with SPSS statistical software (version 13.0; IBM Corporation, Armonk, NY). All the possible risk factors associated with early mortality were screened with $\chi^{2}$ tests for categoric covariates and $t$ tests or Wilcoxon rank sum tests for continuous covariates. Those variables with $P$ values greater than .2 were excluded. A stepwise multivariate logistic regression was then performed with a backward method (criteria for entry and removal of variables were $P<.05$ and $P>$ $.10)$, with 1 variable eliminated at a time. Stability of the model was checked every time a variable was eliminated. When all statistically nonsignificant variables had been eliminated, Hosmer-Lemeshow $\chi^{2}$ test was used to evaluate the calibration and the area under the receiver operating characteristic curve was used to assess how well the model could discriminate between patients who lived and patients who died. Multicollinearity of the identified risk factors was checked with tolerance and inflation statistics.

Informed consent was provided by participants. This study was approved by the local institutional review board and ethics committee (institutional review board and ethics committee of Fuwai hospital, approval number 219).

\section{RESULTS}

In all, 43 surgical centers from 17 province-level regions in mainland China participated in the project, giving a total of 9838 consecutive adult patients. After imputation of the missing variables, all 9838 patients were entered into our analysis. Elective procedures accounted for $97.1 \%$, isolated CABG accounted for $87.7 \%$, and off-pump procedures accounted for $52.4 \%$. Overall in-hospital mortality was $2.5 \%$.

\section{Risk Profiles}

Multivariate analysis identified 11 risk factors as being related to in-hospital mortality: age (continuous), body mass index (continuous), left ventricular ejection fraction (continuous), preoperative New York Heart Association functional class III or IV, chronic renal failure, extracardiac arteriopathy, chronic obstructive pulmonary disease, preoperative atrial fibrillation or flutter (within 2 weeks), preoperative critical state, other than elective surgery, and combined valve procedure. The definitions and statistical characteristics of the risk profiles are listed in Table 1. Multicollinearity diagnostics with tolerance statistics excluded multicollinearity of the 11 factors.

The calibration of the model was satisfactory $(P=.35$ with Hosmer-Lemeshow), and the discrimination power was good (area under the receiver operating characteristic curve, $0.81 ; 95 \%$ confidence interval, $0.79-0.84$ ).

\section{Clinical Outcomes}

The overall in-hospital mortality (defined as any inhospital death) was $2.5 \%$. For patients undergoing elective procedures, the in-hospital mortality was $2.3 \%$ while the mortality rate was $11.1 \%$ for patients undergoing nonelective surgeries (Definition of nonelective surgery was as specified in Appendix E2). The mortality rate for isolated-CABG was $2.0 \%$, and the rate increased to $6.3 \%$ when $\mathrm{CABG}$ was combined with valve procedures. For patients without any identifiable risk factors (whether significant or not), the baseline mortality was $0.5 \%$.

\section{DISCUSSION}

\section{Necessity of First Large-Scale Study in China}

In a phenomenon known as the epidemiologic transition. ${ }^{1}$ the incidence of coronary artery disease in China is increasing with an unprecedented speed. It is estimated to be 98 per 100,000 and may further increase by more than $50 \%$ in the next 2 decades. ${ }^{4}$ This foreshadows an enormous disease burden in the world's most populous country, with 


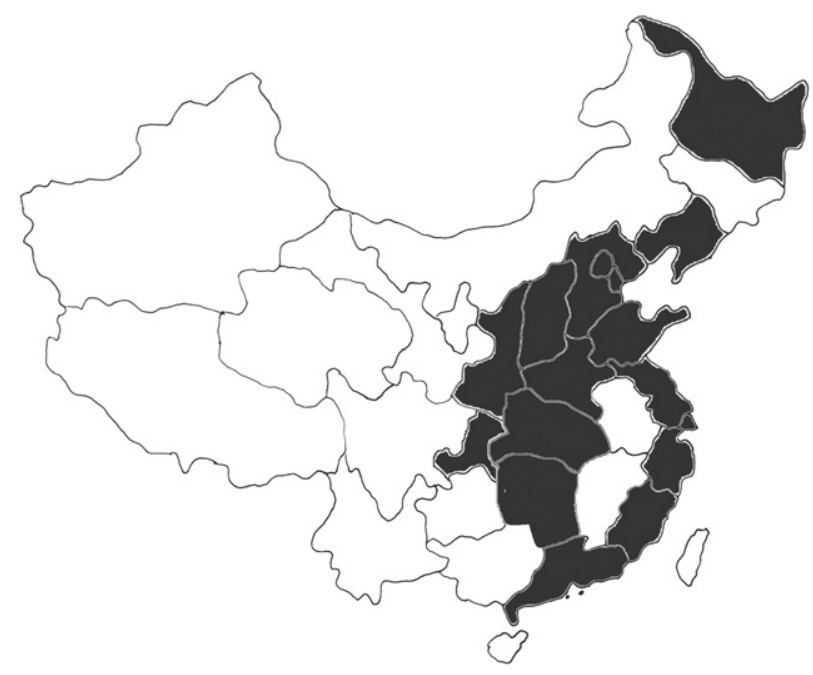

FIGURE 1. Distribution of institutions participating in this registry.

currently more than 1.3 billion people. Parallel to the increase of patients with coronary artery disease, CABG procedures have also shown a dramatic increase. In 1992, the total number of $\mathrm{CABG}$ procedures all over China was fewer than $500,{ }^{5}$ whereas in 2008 , more than 5000 CABG procedures were carried out in 17 institutions of our registry. Despite this rapid growth in surgical procedures, little is known about the current risk profiles of Chinese patients undergoing CABG. There have been attempts to use the "readymade" experience from the published literature derived from large databases of Western countries. Databases like that of the Society of Thoracic Surgeons ${ }^{6}$ are proprietary, however, and other existing models, such as the EuroSCORE ${ }^{7}$ have failed to provide accurate risk assessments in a cohort of Chinese patients undergoing $\mathrm{CABG},{ }^{3}$ suggesting different risk profiles in the Chinese population than for

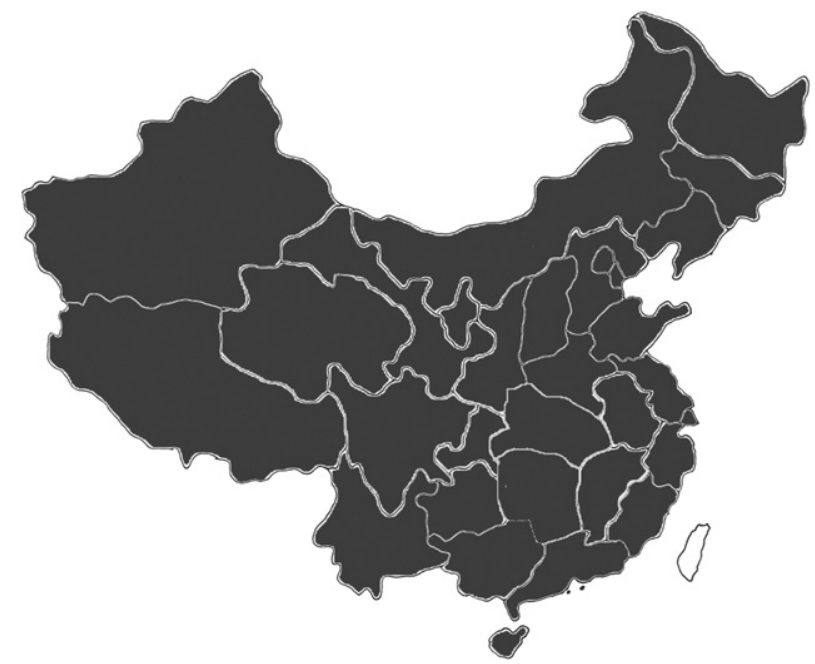

FIGURE 2. Distribution of patients from different province-level regions of China included in this registry.
TABLE 1. Risk factors for in-hospital mortality in Chinese patients undergoing CABG

\begin{tabular}{lccr}
\hline \multicolumn{1}{c}{ Variable } & $\begin{array}{c}\text { Odds } \\
\text { ratio }\end{array}$ & $\begin{array}{c}\mathbf{9 5} \% \text { Confidence } \\
\text { interval }\end{array}$ & $\begin{array}{c}\boldsymbol{P} \\
\text { value }\end{array}$ \\
\hline Age (continuous) & 1.06 & $1.04-1.08$ & $<.001$ \\
Body mass index (continuous) & 0.91 & $0.87-0.96$ & $<.001$ \\
$\begin{array}{l}\text { Left ventricular ejection fraction } \\
\quad \text { continuous) }\end{array}$ & 0.97 & $0.95-0.98$ & $<.001$ \\
$\quad$ New York Heart Association class & 1.92 & $1.43-2.58$ & $<.001$ \\
$\quad$ III or IV & & & \\
Chronic renal failure & 2.40 & $1.27-4.53$ & .007 \\
$\begin{array}{l}\text { Extracardiac arteriopathy } \\
\text { Chronic obstructive pulmonary }\end{array}$ & 4.38 & $2.30-8.33$ & $<.001$ \\
$\quad 2.46$ & $1.36-4.45$ & .003 \\
$\quad$ disease & & & \\
Preoperative atrial fibrillation or & 1.97 & $1.33-2.92$ & .001 \\
$\quad$ flutter & & & \\
Preoperative critical stage & 2.20 & $1.29-3.75$ & .004 \\
$\quad \begin{array}{l}\text { Nonelective surgery } \\
\text { Combined valve surgery }\end{array}$ & 2.38 & $1.30-4.35$ & .005 \\
\hline
\end{tabular}

"more mature" CABG populations in the developed world. Identification of the risk factors for contemporary Chinese patients undergoing CABG is thus a prerequisite for informed consent by patients and for the assessment of the quality of care among different institutions, which might aid in the allocation of medical resources.

Eleven variables were identified as independent risk factors for in-hospital mortality among Chinese patients undergoing CABG. Most of these factors had been described by other studies, ${ }^{8,9}$ such as age and chronic renal failure; however, at least 2 important differences between our population and other databases were observed. First, the percentage of off-pump surgery was very high in our cohort of patients $(52.4 \%)$. Of all the off-pump procedures, most of them $(98.3 \%)$ were carried out on the basis of the doctor's decision. We have noticed that the percentage of off-pump CABG was much lower in Western population (eg, in US, the rate was $20 \%$ in $2006^{10}$ ), and our database contained higher rate of off-pump procedures, reflecting the preference and confidence of Chinese surgeons for off-pump techniques in contemporary China. This may be attributable to technique advances (eg, anesthesia, myocardial protection) achieved in recent years and to prolonged training required for cardiovascular surgeons before one could carry out CABG procedures in the majority of centers of this registry. ${ }^{11}$ Second, reoperation (previous cardiac surgery or previous $\mathrm{CABG}$ ), was not a significant risk factor for in-hospital death after CABG in our patients, although most other non-Chinese studies have included it as a heavily weighted risk factor. ${ }^{7,9,12,13}$ This may be due to 2 reasons: (1) patients who had previous cardiac surgery accounted for very small portion of the whole cohort (previous cardiac surgery, $1.1 \%$; previous $\mathrm{CABG}, 0.3 \%$ ), and (2) these patients often received care in the best heart centers in 
China. In our database, all the patients who had previous CABG history underwent reoperation in Beijing, Shanghai, or Tianjin (direct-controlled municipalities with the best medical resources in this country); of them, $40 \%$ of patients received care from China's largest heart center, Fuwai Hospital. In addition, these low reoperation rates may not only reflect the current situation in China but also be representative for other developing countries where coronary artery disease is becoming increasingly prominent.

Our multi-institutional database, which is based on patients from all over China (a country with 1 fifth of the world's population), is a pioneering attempt to explore the risk factors and to describe the clinical outcomes in the Chinese CABG population. Moreover, to our knowledge, this is also among the first attempts of its kind in Asia, which nurtures more than 2 thirds of the world's population. Conducted at exactly the point of epidemiologic transition, this study may well demonstrate the risk profiles in a less mature $\mathrm{CABG}$ population relative to those in the developed world. $\mathrm{CABG}$ is a relatively recent and growing procedure in China and other developing countries.

This large multicenter database has provided useful information concerning the risk profiles and clinical outcome in Chinese heart centers. As the very first of its kind and with its up-to-date data, our study would help Chinese surgeons with better evaluation of their patients, help Chinese patients with clearer informed consent, and help politicians to allocate valuable medical resources better. In addition, we hope that our experience could be further applied to other Asian populations (such as India) that are now going through the same problem of epidemiologic transition as China.

\section{Limitations}

Three major limitations are noted in this study: First, this study used in-hospital mortality as the major end point, whereas the science of outcome analysis has recommended a longer interval (eg, 6 months) for a more accurate evaluation of early risk for patients undergoing CABG. ${ }^{14}$ Longer follow-up of patients is therefore needed in further studies to delineate more accurate and complete risk profiles for Chinese patients undergoing CABG.

Second, this study, conducted at the point of epidemiologic transition, would probably be out of date in the near future, as previous studies have demonstrated that risk profiles of coronary artery operations change with time. ${ }^{15}$ The characteristics of Chinese patients undergoing CABG are changing rapidly, with more complicated clinical scenarios arising (such as growing rates of reoperation). Because most other studies have focused on more mature CABG populations, our study on a less mature patient cohort may contribute to the literature dealing with the increasing numbers of patients in exactly this less mature population. Moreover, a baseline graph was portrayed at this "preexplosion" moment, which may help in the future.
Third, because this study was based on an exclusively Chinese population and was validated with the same data set, great caution should be taken if our experience is to be applied to other populations. Nevertheless, it may still be of some importance for populations that are at the point of epidemiologic transition.

\section{CONCLUSIONS}

The risk profiles and clinical outcomes of Chinese patients undergoing CABG were analyzed with data from the most up-to-date multi-institutional database. Eleven variables were demonstrated to be independent risk factors for post-CABG in-hospital death.

This study was conducted on behalf of the Chinese CABG Registry Study. The consultant surgeons involved are (in alphabetical order by family name) Haisheng Chen, Liangwan Chen, Xin Chen, Yifan Chi, Zhaoyun Cheng, Nianguo Dong, Tianxiang $\mathrm{Gu}$, Jianguo Hu, Shengshou Hu, Tao Han, Shulin Jiang, Ye Kong, Jiali Liang, Jianshi Liu, Su Liu, Yingze Li, Xiaocheng Liu, Zhiyong Liu, Chengchao Sun, Peixiong Su, Liang Tao, Chuzhong Tang, Fenglin Wang, Junsheng Wang, Chunsheng Wang, Mingying Wu, Ruobin Wu, Shuming Wu, Dong Xu, Feng Xiao, Jiyan Xie, Mingdi Xiao, Yingbin Xiao, Ping Xu, Xiangming Xu, Zhiyun Xu, Song Xue, Biao Yuan, Chuanrui Yang, Dinghua Yi, Qiang Zhao, Kaiguang Zhang, Shunye Zhang, Wenjun Zhen, and Chengwei Zou. We acknowledge the assistance of each center listed here for collecting and validating the data. We also thank the Chinese CABG Registry Study database working staff (Yan Zhao, Huan Ren, Na Tian, and Zhiming Zhu) for their industrious efforts. The 43 centers (in alphabetic order) participating in the Chinese CABG registry study (2007-2008) were Affiliated Hospital of Jining Medical College, Beijing ChaoYang Hospital, Beijing Friendship Hospital, Beijing Hospital, Beijing Tongren Hospital, Beijing Xuanwu Hospital, Changhai Hospital of The Second Military Medical University, ChinaJapan Friendship Hospital, Fujian Provincial Hospital, Fujian Medical University Union Hospital, Fuwai Hospital, Guangdong Cardiovascular Institute, Guangdong Gaozhou People's Hospital, Henan Anyang People's Hospital, Henan Provincial People's Hospital, Jiangsu People's Hospital, Nanjing Firtst Hospital, Peking University First Hospital, Peking University People's Hospital, Ruijin Hospital of Shanghai Jiao Tong University, Shandong University Qilu Hospital, Shandong Provincial Hospital, Shanghai Renji Hospital, Shanghai Chest Hospital, Shanghai First People's Hospital, Shanxi Cardiovascular Hospital, Teda International Cardiovascular Hospital, The Affiliated Hospital of Medical College Qingdao University, The First Hospital of China Medical University, The First Affiliated Hospital of Wenzhou Medical College, The General Hospital of Jinan Military region, The Second Affiliated Hospital of Harbin Medical University, The Second Hospital of Hebei Medical University, The Second Xiangya Hospital of Central South University, Tianjin Chest Hospital, Tongji Medical College of Huazhong University of Science and Technology Union Hospital, Wuhan Asia Heart Hospital, Xijing Hospital of The Fourth Military Medical University, Xinqiao Hospital of The Third Military Medical 
University, Xuzhou Central Hospital, Zhongda Hospital, Southeast University, Zhongshan Hospital Fudan University.

\section{References}

1. Okrainec K, Banerjee DK, Eisenberg MJ. Coronary artery disease in the developing world. Am Heart J. 2004;148:7-15.

2. Hannan EL, Siu AL, Kumar D, Racz M, Pryor DB, Chassin MR. Assessment of coronary artery bypass graft surgery performance in New York. Is there a bias against taking high-risk patients? Med Care. 1997;35:49-56.

3. Zheng Z, Li Y, Zhang S, Hu S, Chinese CABG Registry Study. The Chinese coronary artery bypass grafting registry study: how well does the EuroSCORE predict operative risk for Chinese population? Eur J Cardiothorac Surg. 2009;35: 54-8.

4. Moran A, Gu D, Zhao D, Coxson P, Wang YC, Chen CS, et al. Future cardiovascular disease in China: Markov model and risk factor scenario projections from the coronary heart disease policy model-China. Circ Cardiovasc Qual Outcomes. 2010;3:226-7.

5. Zhang H, Hu S. [History of the grafting of coronary artery bypass]. Zhonghua $\mathrm{Yi}$ Shi Za Zhi. 2001;31:148-52. Chinese.

6. Edwards FH, Grover FL, Shroyer AL, Schwartz M, Bero J. The Society of Thoracic Surgeons National Cardiac Surgery Database: current risk assessment. Ann Thorac Surg. 1997;63:903-8.

7. Nashef SA, Roques F, Michel P, Gauducheau E, Lemeshow S, Salamon R. European system for cardiac operative risk evaluation (EuroSCORE). Eur J Cardiothorac Surg. 1999;16:9-13.
8. Parsonnet V, Dean D, Bernstein AD. A method of uniform stratification of risk for evaluating the results of surgery in acquired adult heart disease. Circulation. 1989;79(6 Pt 2):I3-12. Erratum in: Circulation. 1990;82:1078.

9. Eagle KA, Guyton RA, Davidoff D, Ewy GA, Fonger J, Gardner TJ, et al. ACC/ AHA guidelines for coronary artery bypass graft surgery: executive summary and recommendations: A report of the American College of Cardiology/American Heart Association Task Force on Practice Guidelines (Committee to revise the 1991 guidelines for coronary artery bypass graft surgery). Circulation. 1999;100:1464-80.

10. Puskas JD, Steele M. Would you like some cardiopulmonary bypass with your coronary revascularization? Circulation. 2007;116:1756-8.

11. Hu S, Zheng Z, Yuan X, Wang W, Song Y, Sun H, Xu J. Increasing long-term major vascular events and resource consumption in patients receiving off-pump coronary artery bypass: a single-center prospective observational study. Circulation. 2010;121:1800-8.

12. Reid C, Billah B, Dinh D, Smith J, Skillington P, Yii M, et al. An Australian risk prediction model for 30-day mortality after isolated coronary artery bypass: the AusSCORE. J Thorac Cardiovasc Surg. 2009;138:904-10.

13. Hannan EL, Wu C, Bennett EV, Carlson RE, Culliford AT, Gold JP, et al. Risk stratification of in-hospital mortality for coronary artery bypass graft surgery. $J$ Am Coll Cardiol. 2006;47:661-8.

14. Osswald BR, Blackstone EH, Tochtermann U, Thomas G, Vahl CF, Hagl S. The meaning of early mortality after CABG. Eur J Cardiothorac Surg. 1999;15: 401-7.

15. Miller DC, Stinson EB, Oyer PE, Jamieson SW, Mitchell RS, Reitz BA, et al Discriminant analysis of the changing risks of coronary artery operations: 1971-1979. J Thorac Cardiovasc Surg. 1983;85:197-213. 


\section{APPENDIX E1. SIXTY-SEVEN PREOPERATIVE RISK FACTORS FOR UNIVARIATE ANALYSIS}

Sex, age (in years), height (in meters), weight (in kilograms), body mass index (in kilograms per square meter), body surface area (in square meters), history of smoking, family history of coronary artery disease (immediate family members with diagnosed coronary artery disease or sudden cardiac death before 55 years of age), diabetes mellitus, hyperlipidemia (total cholesterol $>5.18 \mathrm{mmol} / \mathrm{L}$, low-density lipoprotein $\geq 3.37 \mathrm{mmol} / \mathrm{L}$, high-density lipoprotein $<0.78$ $\mathrm{mmol} / \mathrm{L}$, or triglycerides $>1.69 \mathrm{mmol} / \mathrm{L}$ ), hypertension, chronic renal failure (documented history or any previous serum creatinine level $>176 \mu \mathrm{mol} / \mathrm{L}$ ), cerebrovascular accident (documented history of coma $\geq 24$ hours or central nervous system dysfunction $\geq 72$ hours), endocarditis (documented history or diagnosis with blood culture and cardiac ultrasonography), chronic obstructive pulmonary disease (long-term use of bronchodilators or steroids for lung disease), immunosuppressive therapy (within 30 days before surgery), extracardiac arteriopathy (any 1 or more of the following: claudication, carotid occlusion or $>50 \%$ stenosis, previous or planned intervention on the abdominal aorta, limb arteries, or carotids), previous cardiac surgery, previous coronary artery bypass grafting, previous valve surgery, previous percutaneous balloon valvuloplasty, previous percutaneous coronary intervention, previous permanent pacemaker placement, previous history of noncardiac surgery, history of myocardial infarction, recent myocardial infarction ( $<21$ days), heart failure (within 2 weeks), unstable angina (rest angina requiring intravenous nitrates until arrival in the anesthetic room), cardiogenic shock (lasting until arrival in the anesthetic room), cardiopulmonary resuscitation (within 2 hours), preoperative persistent ventricular tachycardia or fibrillation (within 2 weeks), preoperative III atrioventricular block (within 2 weeks), preoperative atrial fibrillation or flutter (within 2 weeks), preoperative critical stage (any 1 or more of the following: preoperative cardiogenic shock, ventricular fibrillation or flutter, preoperative intra-aortic balloon pump implantation), New York Heart Association functional class, Killip staging (for patients with acute myocardial infarction), Canadian Cardiovascular Society class (for patients with angina), serum creatinine, total cholesterol, triglycerides, low-density lipoprotein, high- density lipoprotein, hemoglobin, high-sensitivity C-reactive protein, erythrocyte sedimentation rate, left carotid artery lesion, right carotid artery lesion, left renal artery stenosis, right renal artery stenosis, number of involved coronary arteries, left main stenosis, left descending stenosis, circumflex stenosis, right coronary stenosis, graft stenosis, left ventricular ejection fraction (assessed by echocardiography at the last time before surgery), ventricular aneurysm, pulmonary hypertension (systolic pulmonary arterial pressure $>60 \mathrm{~mm} \mathrm{Hg}$ ), valve stenosis or insufficiency, aortic stenosis, mitral stenosis, tricuspid stenosis, pulmonary stenosis, aortic insufficiency, mitral insufficiency, tricuspid insufficiency, pulmonary insufficiency

\section{APPENDIX E2. THIRTY OPERATIVE RISK FACTORS FOR UNIVARIATE ANALYSIS}

Nonelective surgery (Emergency or salvage surgery, with emergency surgery defined as unscheduled surgery required on the same day because of refractory angina despite maximal medical therapy, acute evolving myocardial infarction within 24 hours before surgery, aortic dissection, percutaneous coronary intervention failure, or other reasons, and salvage surgery defined as operations on patients who had cardiopulmonary resuscitation before arrival in the anesthetic room), on-pump surgery, time of extracorporeal circulation, time of aortic crossclamping, beating-heart surgery (on-pump surgery with ventricular fibrillation is considered beating-heart surgery), site of cannula, aortic crossclamp, intra-aortic balloon pump support during or after coronary artery bypass grafting, ventricular assistance device, heart transplant, extracorporeal membrane oxygenation, number of grafts, combined valve surgery (surgical procedures with either valve), procedure on aortic valve, procedure on mitral valve, procedure on tricuspid valve, procedure on pulmonary valve, repair for ventricular aneurysm, repair of septal rupture, excision of heart tumor, repair of congenital heart defects, laser drilling, repair of injured cardiac muscle, implantation of permanent pacemaker, surgery for atrial fibrillation, procedure on thoracic aorta, cell transplant, hybrid procedure (planned hybrid procedure with percutaneous coronary intervention and coronary artery bypass grafting), carotid procedure, postinfarction septal rupture 